\title{
Delivering Sprinkles Plus through the Integrated Child Development Services (ICDS) to Reduce Anemia in Pre-school Children in India: Author's Reply
}

\author{
Siddhivinayak Hirve
}

Received: 11 February 2014 / Accepted: 12 March 2014 /Published online: 29 March 2014

(C) Dr. K C Chaudhuri Foundation 2014

To the Editor: We thank Kapil et al. for their critique on our paper for not presenting data on hemoglobin increase, compliance, side-effects, and cost-effectiveness of Sprinkles and question the use of Hemocue to estimate hemoglobin [1].

We agree that presenting details on hemoglobin change would have been more informative. In our study, pre- and post-intervention hemoglobin levels were estimated in two independent cross-sections sampled from a larger cohort of children who received the intervention. This precludes us from estimating change in hemoglobin levels. Instead we present the reduction in proportion of anemic children as a meaningful program evaluation outcome.

We have previously presented information on Sprinkles side-effects [2]. The current study was designed in the context of an Integrated child development services (ICDS) pilot intervention program to evaluate delivery and impact of Sprinkles on anemia. We intended ICDS itself to measure hemoglobin, compliance and side-effects to encourage ownership. The ICDS worker over-ascertained the compliance. The postintervention survey did not have enough power to test differences in compliance by age groups or by place of intervention. It was agreed that the ICDS will only monitor side-effects that result in prolonged interruption or discontinuation of Sprinkles. Furthermore, it was prone to varying recall bias since the survey took place after the 4-mo intervention period while the majority of children had completed receiving Sprinkles in the first 2 mo itself.

\footnotetext{
S. Hirve $(\square)$

Vadu Rural Health Program, KEM Hospital Research Center, Rasta Peth, Pune 411011, India

e-mail: sidbela@vsnl.com
}

It was beyond the scope of this paper to evaluate the costeffectiveness of Sprinkles. It was supplied to ICDS by the manufacturer at no cost.

Hemoglobin can be measured on a variety of devices that use different principles of operation. Furthermore, the clinical measurement of total hemoglobin has inherent variability. CO-Oximeters use spectrophotometric detection and are considered the gold standard for hemoglobin measurement. Its accuracy is a function of multiple variables related to the device as well as collection and handling of blood analyte that result in significant intra- and inter-device variation (up to $1.2 \mathrm{~g} / \mathrm{dL}$ ) in the hemoglobin measurements. Use of point-of-care analyzers based on spectrophotometry detection (e.g., HemoCue) is widely accepted in critical care, bedside and field survey settings because of quick results with portable devices and smaller samples from capillary puncture.

Conflict of Interest None.

Role of Funding Source This work was supported by Helen Keller International and HJ Heinz Foundation.

\section{References}

1. Hirve S, Martini E, Juvekar SK, Agarwal D, Bavdekar A, Sari M, et al. Delivering sprinkles plus through the integrated child development services (ICDS) to reduce anemia in pre-school children in India. Indian J Pediatr. 2013;80:990-5.

2. Hirve S, Bhave S, Bavdekar A, Naik S, Pandit A, Schauer C, et al. Low dose 'Sprinkles'-An innovative approach to treat iron deficiency anemia in infants and young children. Indian Pediatr. 2007;44:91-100. 